\title{
Effect of Myoinositol (MI) In Patients of Polycystic Ovarian Disease (PCOD)
}

\author{
Authors
}

\section{Dr Jayshree Awalekar ${ }^{1}$, Dr Chidanand Awalekar', Dr M. H. Patwardhan ${ }^{3}$}

${ }^{1}$ M.D, Professor, Dept of Medicine, Bharati Vidyapeeth Deemed University Medical College \& Hospital, Sangli

Email: jaysri2065@gmail.com, Mobile:09422045082 Fax No. : 0233-2601201

${ }^{2}$ M.D, Professor \& Head Of Dept, Medicine, Bharati Vidyapeeth Deemed University Medical College \& Hospital, Sangli

Email: awalekar11cd7@gmail.com, Fax No. : 0233-2601201, Mobile: 09422045081

${ }^{3}$ M.D.D.M. (Endocrinology), Patwardhan Endocrinology Clinic \& Research Centre, Miraj

Email: rajapatwardhan@yahoo.co.in, Mobile:09860615285 Fax No. :0233-2601201

\section{Abstract}

Polycystic ovarian disease (PCOD) is a common endocrine disorder. Majority of young females are affected. Insulin resistance along with dysfunction of hypothalamo pituitory adrenal axis is a key etiological factor in development of all manifestations of PCOD. They present with irregular menses, infertility, obesity, hirsutism, acanthosis Nigricans etc. MI(MI), an insulin sensitizer is widely accepted treatment modality. Data suggests variable responses to MI. So we aimed to study efficacy of MI in PCOS. Here 32 cases are recruited those diagnosed by using Rotterdam's criteria. BMI, menstrual irregularity, infertility, hirsutism, acanthosis Nigricans, acne were common presenting features. Hormonal \& biochemical profile was studied S.LH (Luteinizing Hormone), FSH (Follicle stimulating hormone), Serum. Prolactin levels, Serum Insulin levels were studied. Weight loss, menstrual regularity, LH/FSH Ratio, HOMA Index was studied initially \& compared all parameters after 3 months of treatment. In our study BMI mean was 26.71, after MI treatment it became 25.6.Differences of all parameters before \& after MI suggests, there is significant reduction in BMI \& LH/FSH ratio. Other parameters are not affected by MI. It suggests MI has been effective in reducing BMI \& LH/ FSH ratio significantly. But there was no change in HOMA index. So MI can be good supportive treatment option in PCOD.

The present study gives us idea about efficacy of MI treatment in PCOD cases \& will be useful in patient treatment schedules.

Keywords- PCOD (Polycystic ovarian Disease), Hyperinsulinemia, Myoinositol (MI), Body Mass Index (BMI), LH/FSH-Luteinizing hormone \& Follicle stimulating hormone ratio, HOMA index-Homeostatic model assessment.

\section{Introduction}

Nowadays Poly Cystic Ovarian Disease (PCOD) is commonest endocrine disorder in young females. It is characterised by insulin resistance \& is strongly implicated in its aetiology.

"Polycystic ovary syndrome: a complex condition with psychological, reproductive and metabolic manifestations that impacts on health across the lifespan". (2) In 2003 a consensus workshop sponsored by ESHRE/ASRM in Rotterdam indicated PCOS to be present if any 2 out of 3 criteria are met ${ }^{(2,7)}$ it also includes many women without androgen excess too. Rotterdam's criteria are widely accepted. 
1) Oligoovulation and/or anovulation.

2) Excess androgen activity.

3) Polycystic ovaries (by ultrasound).

4) Other entities are excluded that would cause these. ${ }^{(5,7)}$

PCOD patients present with menstrual irregularities like amenorroea, oligomenorrhoea about half of them have infertily because of anovulation caused by hormonal dysfunction \& insulin resistance. Hirsutism, acne, obesity, miscarriages, hypertension, Ischaemic Heart Disease, diabetes, sleep apnoea etc ${ }^{(2,3,8)}$.

The population studies revealed first, that overt and occult PCOD accounted for $90 \%$ of patients with oligomenorrhea and $37 \%$ with amenorrhea, or $73 \%$ with oligo or amenorrhea. Oligo or amenorrhea accounted for $21 \%$ of couples with infertility and the annual incidence was 247 patients per million of the general population. The annual incidence of infertility due to PCOD per million was 41 with overt PCOD and 139 with occult PCOD (total 180). Of those, 140 appeared to respond well to clomiphene $(78 \%)$ but 40 (22\%) failed, requiring alternative therapy ${ }^{(4,5)}$

Insulin resistance \& obesity is observed in majority of PCOD patients. Elevated insulin levels cause abnormal functioning of hypothalamic-pituitary-ovarian axis that lead to PCOS. Women with PCOS experience an increased frequency of hypothalamic $\mathrm{GnRH}$ pulses, which in turn results in an increase in the LH/FSH ratio ${ }^{(4,5,8)}$.

Insulin resistance is main causative factor for all these consequences $\&$ morbidity. Failure of the target cells to respond to normal or ordinary levels of insulin is regarded as insulin resistance irrespective of the body mass index (BMI). Hyperinsulinaemia due to insulin resistance occurs in approximately $80 \%$ of PCOS women with central obesity \& 30\%-40\% of lean PCOS women. Measurement of the fasting insulin concentration $^{(10)}$ is an easy marker of insulin resistance. S. Insulin levels of 20 or higher indicates that insulin resistance is present. Hyperinsulinemia is the main causative factor in PCOD women both obese \&lean ${ }^{(4,5)}$ \& cause hyperandrogenism. Insulin directly promotes ovarian steroidogenesis, and inhibits liver release of the sex hormone binding globulin (SHBG) and production of insulin-like growth factor binding protein 1 (IGFBP-1). Increased concentrations of IGF-1 additionally promote ovarian release of androgens ${ }^{(1,4,7)}$.

\section{Insulin Resistance}

We have used HOMA index as a marker of insulin resistance, based on measurements of fasting glucose and insulin levels, is the homeostatic mode assessment (HOMA-IR). Resistance to insulin is diagnosed at HOMA-IR levels $\geq 3.8^{(1,4)}$. Insulin resistance can manifest as follows:

1. Insulin resistance/Type II diabetes ${ }^{(1,4,9,11)}$.

2. Weight gain ${ }^{(1,3)}$

3. High blood pressure, in obese and/or during pregnancy. ${ }^{(1,5,7)}$

4. Cardiovascular disease-two fold increased risk of arterial disease in PCOS patients as compared to women without PCOS ${ }^{(7,11)}$

5. Strokes ${ }^{(2,3)}$

6. Miscarriage ${ }^{(6,7)}$

7. Sleep apnoea, in obese $\mathrm{e}^{(7,11)}$

8. Non-alcoholic fatty liver disease, in obese $^{(11)}$

9. Acanthosis nigricans (patches of darkened skin under the arms, in the groin area, on the back of the neck ${ }^{(1,6,11)}$.

Adipose tissue enzyme, aromatase, converts androstenedione to estrone and testosterone to estradiol. The excess of adipose tissue in obese led excess androgen formation, which are responsible for hirsutism and virilization Excess estrogens inhibits FSH via negative feedback ${ }^{(15)}$. It is been treated with various treatment modalities successfully.

MI (MYO) and D-chiro- inositol (DCI); both stereoisomers were used, as insulin sensitizer drugs, in the treatment of PCOS treatments ${ }^{(1,4,11)}$. Inositol belongs to the vitamin $\mathrm{B}$ complex. Epimerization of the six hydroxyl groups of inositol leads to the formation of up to nine stereoisomers, including Human adults consume 
approximately $1 \mathrm{~g}$ of inositol (mainly MI) per day in different biochemical forms ${ }^{(4,7,11)}$.

Inositol phosphoglycans (IPGs) activate enzymes that control glucose metabolism ${ }^{(6,11)}$. Defect in the IPGs, second messenger can cause impaired insulin metabolism ${ }^{(1,11)}$. In PCOS women, a defect in tissue availability or altered metabolism of IPGs (inositol) mediators may contribute to insulin resistance ${ }^{(10,11)}$. Circulating free MYO is taken up by most tissues by a membraneassociated sodium- dependent inositol cotransporter; inositol uptake is inhibited by glucose $^{(4,7)}$. In particular, it was shown that MYO had 10 times more affinity for the transporter compared to $\mathrm{DCI}^{(11)}$.

PCOS is associated with selective increase in urinary clearance of D-chiro-inositol (DCI) \& impaired DCI inositol phosphoglycan release in response to insulin. That means defect in tissue availability or utilization of DCI in PCOs that may contribute to insulin resistance.

DCI increases the action of insulin in patients of PCOS, thereby improving ovulatory function \& decreasing serum androgen concentration, blood pressure \& plasma triglyceride concentration. Women with PCOS have Insulin Resistance with hyperinsulinemia because of deficiency of DCI containing phosphoglycan that mediates action of insulin $^{(3,4)}$.

Elevated concentrations of MYO in human follicular fluid play a role in follicular maturity and provide a marker of good-quality oocytes ${ }^{(4,6)}$. Previous studies have demonstrated that MI is capable of restoring spontaneous ovarian activity, and consequently fertility, in most patients with $\operatorname{PCOS}^{(6)}$.

\section{Study Design, Size \& Duration}

This is comparative observational was study done at Bharati Vidyapeeth Deemed University Medical College \& Hospital, Sangli \& Dr. Patwardhan's Endocrinology \& Research Centre Miraj. It was carried out from June 2013 to June 2015. In cases of PCOD effect of MI is observed. We recruited 32 patients of PCOD for this study.
Criteria of Inclusion- All diagnosed cases by using to Rotterdam criteria.

\section{Criteria of Exclusion-}

1. Cushing's syndrome

2. Thyroid disorders

3. Pituitary tumours

4. Diabetes Mellitus

A. Written consent is taken before starting examination.

- History \& Clinical examination is done.

Following points were considered for comparison.

1. Height in metre, weight in $\mathrm{Kg} \&$ Body Mass Index(BMI) is calculated.

2. Menstrual irregularities are noted as amenorrhoea, oligomennorrhoea \& irregular cycles.

3. Infertility.

4. Hirsutism, unwanted hair growth, acanthosis Nigricans - The features of excess androgen levels is noted.

B. Following investigations were done-

1) LH (Luteinizing Hormone).

2) FSH (Follicle stimulating hormone).

3) Serum. Prolactin levels-

4) Serum Insulin levels-

C. Following parameters were studied for comparison

1. BMI reduction-

2. LH/FSH Ratio-

3. HOMA Index-homeostatic model assessment (HOMA) -method used to quantify IR \& beta-cell function. It also predicts cardiometabolic risk .Calculated by using formula HOMA-IR = [Glucose] x [Insulin] / 405 (Glucose in mg/dl.

\section{Study Procedure}

We have included 32 patients of PCOD in this group. Tab MI 2 gm BID along with Tab. Folic acid $5 \mathrm{mg}$ OD is advised to these patients. All parameters of comparison given above repeated after 3 months. 
RESULTS \& DISCUSSION-

MI Effect Before(pre) \& After(post) Treatment-

\begin{tabular}{|l|l|l|l|l|l|}
\hline & Mean & $\begin{array}{l}\text { Std. } \\
\text { Deviation }\end{array}$ & $\begin{array}{l}\text { Std. Error } \\
\text { Mean }\end{array}$ & T & p value \\
\hline pre BMI & 25.40 & 6.53 & 1.15 & 2.77 & $\mathbf{0 . 0 0 9}$ \\
\hline post BMI & 24.40 & 5.91 & 1.04 & & \\
\hline pre HOMA index & 23.74 & 25.00 & 4.42 & 0.006 & 0.995 \\
\hline post HOMA index & 23.8 & 44.62 & 7.88 & & \\
\hline Pre LH/ FSH & 2.32 & 0.29 & 0.05 & 3.78 & $\mathbf{0 . 0 0 1}$ \\
\hline Post LH /FSH & 2.10 & 0.43 & 0.077 & & \\
\hline Pre prolactin & 19.73 & 12.75 & 2.25 & - & 0.420 \\
\hline Post prolactin & & & & 0.817 & \\
\hline
\end{tabular}

1) BMI before treatment with $\mathrm{MI}$ mean was 25.4 ,reduced to $24.4(\mathrm{p}=0.009)$ after treatment, which is statistically significant.

2) HOMA index before treatment was mean 27.74 after treatment it increased despite of reduction to $23.8(\mathrm{p}=0.995)$, which is statistically not significant. So here HOMA index did not have beneficial effect in PCOD cases.

3) LH/ FSH Ratio were mean 2.32 before treatment. After treatment it reduced to mean $2.10(\mathrm{p}=0.001)$, which is statistically significant .This indicates MI is helpful in correcting hormonal dysfunction of PCOD.

4) Prolactin Levels were mean 19.73 before MI treatment. It increased to $21.69(\mathrm{p}=0.420)$ which is statistically not significant.

\section{Discussion}

There is data suggesting MI is useful to varying extent in cases of PCOD. Improvement in Insulin sensitivity, weight loss, menstrual regularity, ovulation by hormonal dysfunction is studied .

Costantino et al. ${ }^{(5)}$ recruited 42 patients treated with MYO showed there was no change in the fasting plasma insulin and glucose concentration. AUC, for both insulin and glucose, decreased during the oral glucose tolerance. There was no change in the fasting plasma insulin and glucose concentration Ovulation was restored in 16 (69.5\%) women treated with MYO group.

Genazzani AD.et al. studied effect of MI in 20 patients compared with placebo. After 12 weeks of treatment, they found that LH, Prolactin, T, insulin levels, LH/FSH results Were significantly reduced. Insulin sensitivity results were significantly improved. Menstrual cyclicity was restored in all amenorrheic and oligomenorrheic subjects ${ }^{(12)}$.

Gerli et al-The BMI decreased significantly in the MYO group ( $p=0.04)$. No change was observed in the waist-to-hip ratio ${ }^{(4)}$.

Raffone et al. showed that MYO slightly improves pregnancy rate compared to metformin. no side effects were reported.

\section{Conclusions}

MI did better in this study in regard to BMI \& $\mathrm{LH} / \mathrm{FSH}$ ratio but there is not significant change in HOMA index \& prolactin levels. Being physiological component It may be considered one of the supportive option in PCOD treatment schedule.

\section{References}

1. Alemzadeh R, Kichler J, Calhoun M. Spectrum of metabolic dysfunction in relationship with hyperandrogenemia in obese adolescent girls with polycystic ovary syndrome. Eur $\mathrm{J}$ Endocrinol. Jun 2010;162(6):1093-9. 
2. Lobo RA, Carmina E. The importance of diagnosing the polycystic ovary syndrome. Ann Intern Med 2000;132:989-993.

3. Barber TM, McCarthy MI, Wass JA, Franks S. Obesity and polycystic ovary syndrome. ClinEndocrinol (Oxf). Aug 2006;65(2):137-45.

4. Gerli S, Papaleo E, Ferrari A, Di Renzo GC. Randomized, double blind placebocontrolled trial: effects of myo-inositol on ovarian function and metabolic factors in women with PCOS. Eur Rev Med Pharmacol Sci 2007;11:347-354.

5. Costantino D, Minozzi G, Minozzi E, Guaraldi C. Metabolic and hormonal effects of myo-inositol in women with polycystic ovary syndrome: a double-blind trial. Eur Rev Med Pharmacol Sci 2009;13:105-110.

6. Minozzi M, D’Andrea G, Unfer V. Treatment of hirsutism with myo-inositol: a prospective clinical study. Reprod Biomed Online 2008; 17:579-582.

7. Nestler JE, Jakubowicz DJ, Reamer P, Gunn RD, Allan G. Ovulatory and metabolic effects of D-chiro-inositol in the polycystic ovary syndrome.N Engl J Med 1999;340:1314-1320.

8. Unfer V, Carlomagno G, Dante G, Facchinetti F Gynecol Endocrinol. 2012 Feb 1-7. Effects of myo-inositol in women with PCOS: a systematic review of randomized controlled trials.

9. Knowler WC, Barrett-Connor E, Fowler $\mathrm{SE}$, et al; Diabetes Prevention Program Research Group. Reduction in the incidence of type 2 diabetes with lifestyle intervention or metformin. N Engl J Med. 2002;346:393-403.

10. Kitabchi AE, Temprosa M, Knowler WC, et al. Role of insulin secretion and sensitivity in the evolution of type 2 diabetes in the diabetes prevention program: effects of lifestyle Metformin in polycystic ovary syndrome: systematic review and meta-analysis
11. Jonathan $\mathrm{M}$ Lord, Ingrid $\mathrm{H} \mathrm{K}$ Flight, Robert J Norman, professor3 BMJ 2003; 327 doi: http://dx.doi.org/10.1136/bmj.327.7421.95 1 (Published 23 October 2003) Cite this as: BMJ 2003;327:951 intervention and metformin. Diabetes. 2005;54:2404-2414.

12. Genazzani AD, Lanzoni C, Ricchieri F, Jasonni VM. Myo-inositol administration positively affects hyperinsulinemia and hormonal parameters in overweight patients with polycystic ovary syndrome. Gynecol Endocrinol 2008;24:139-144. 